\title{
Guidelines for local research ethics committees
}

\author{
P C E Moodie, T Marshall
}

In August last year, for only the second time, the Department of Health issued guidelines on local research ethics committees. ${ }^{1}$ Initially the department (then the Ministry of Health) only drew hospital authorities' attention to guidelines issued by others concerning research on humans. ${ }^{23}$ Not until 1975 did the department make its own statement, ${ }^{4}$ and the present document is the definitive version of an earlier draft circular $^{5}$ which contained the first departmental words on the subject for 14 years. Has it been worth waiting for? Do the guidelines serve what should be the twin aims of local ethics committees: protecting the public from harm and enhancing the conduct of good scientific research? As lay members of different local ethics committees we are concerned that this document is far from the clear and comprehensive statement that committees had the right to expect.

\section{Appointing authority and responsibility}

As before, the district health authority will appoint the committee, but the committee's responsibility has been extended to reviewing research on humans undertaken by any NHS body - that is, including the family health services authorities, special health authorities, or trusts, as well as NHS patients treated elsewhere. However, the committee now has only an advisory role in relation to the research, and the NHS body itself decides whether the research should proceed. Thus the guidelines firmly state that committees "are not in any sense management arms of the District Health Authority." The summary sheet accompanying the guidelines stated: "Any NHS body asked to approve a research proposal must consult its local research ethics committee." This assumes channels of communication-from the researcher to the NHS body to the committee and back againwhich simply do not exist. Nicholson points to the inevitable delay which will occur if a second level of decision making is introduced encompassing elements (for example, finance, workload) outside those which the committee is supposed to consider. ${ }^{6}$ Who is to make these decisions, on what basis, and what further information will researchers be expected to provide? No information and no guidelines are offered. As Nicholson also points out, this will serve to proliferate inconsistency in decision making between NHS bodies, ${ }^{6}$ behaviour which ethics committees have been criticised for and which the new guidelines would be expected to help minimise.

Faculty of Law and Department of Public Health and Epidemiology, University of Birmingham, Birmingham B15 2TT PC E Moodie, lecturer T Marshall, senior lecturer

Correspondence to: Mr Marshall.

BMF 1992;304:1293-5 ing that the Community Health Council be invited to nominate a lay person to the committee. The new recommendations are for two or more lay persons, plus hospital medical staff, nursing staff, and general practitioners. Both sexes and "a wide range of age groups" should be represented. The guidelines do not specify that hospital medical representatives should be consultants, but that is the current norm. Explicit provision for including junior staff would have been a good way both of broadening the age range and of using their experience in the ethical conduct of research on busy wards.

Several districts have included a lawyer on the committee and others a hospital chaplain. The new guidelines do not mention places being reserved for either profession. This is sensible as their relevant skills are not exclusively held and there would be a risk of a "me too" approach from other professions demanding representation (pharmacists, statisticians, etc). On the other hand, committees must be systematically and authoritatively aware of the legal dimensions of their work - for example, with regard to issues of consent and the implications of compensation arrangements for research subjects. Unfortunately, the guidelines do not mention the need for training of committee members.

The draft circular recommended that a lay person should chair the committee, though without explaining why. This suggestion produced considerable hostility, not least from a distinguished lay member and former minister for health, Sir Kenneth Robinson. ${ }^{7}$ The final document has stepped back somewhat, requiring that at least one of the two posts of chairperson and vice chairperson (the second being a new position) should be filled by a lay member. Again, however, there is no explanation of why. It seems most unlikely that many lay members will be appointed as chairpersons - the technical complexity of many of the proposals we encounter would make such appointments impractical in our districts - though the imperative for a medically qualified chairperson may be less in districts without university and teaching hospitals. On the other hand, the post of vice chairperson seems something of a sop cast in the direction of whatever unexplained principle prompted the original proposal. If the belief was that a lay chairperson would ensure public confidence in the work of local ethics committees, the secrecy of their proceedings denies the premise. Publication of an annual report is the only public face allowed to the committee, and it remains to be seen whether this will convince local media and the Community Health Council that its business has been properly conducted.

\section{Scope of research}

The boundaries of research that need approval by the committee have been considerably extended. For the first time epidemiological research using existing records must be submitted to the committee. The circular acknowledges that it will be difficult to obtain consent from subjects for their medical records to be used in this way and suggests an "opting out" form of consent - if patients have indicated they do not want their records used they should be excluded. But how are patients ever to know about this, unless asked directly? Epidemiologists in general, and public health 


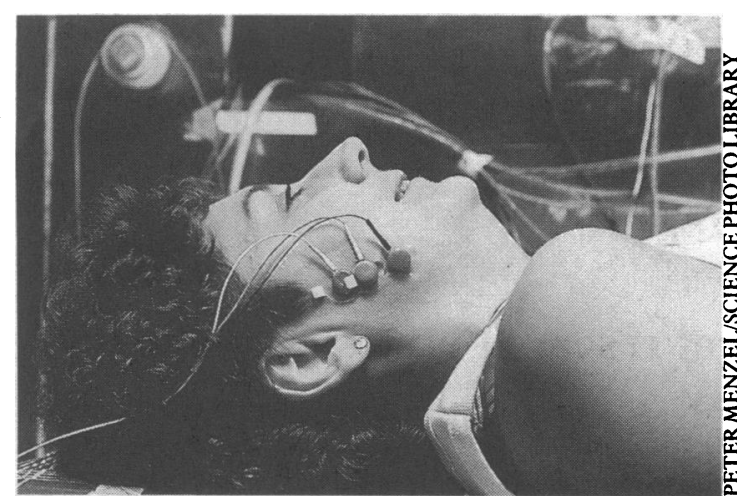

Research subjects must be given appropriate information to allow them to decide whether to participate

doctors in particular, would no doubt welcome further clarification on this matter. Similarly, Smith highlighted a researcher's concern that research using questionnaires will for the first time require committee approval. ${ }^{8}$ However, many committees already expect to see proposals for research by questionnaire because of the risk that some questions may cause anxiety or distress to patients. The Department of Health is therefore wise to insist on a broad remit for ethics committees: the comment Smith reported is a good example of how ethical issues may not be perceived by those engaged in research as a daily activity.

One aspect of research, however, is effectively ignored in the guidelines. The committee is required to bring to the attention of its appointing body any indication that research is being carried out which it has not been asked to consider. Inevitably this obligation will sometimes require a consideration of what constitutes research. Chapter 1 of the guidelines provides a list of the types of research project for which committees must be consulted, but the admittedly difficult borderline between research and innovative treatment is not discussed. There is no shortage of views on the subject. For example, Kennedy and Grubb concluded that "To the extent that the intention is to acquire knowledge and not merely to care for the patient ... innovative therapy should be subject to the same regime of control that attends research properly so-called." It is regrettable that the department offers neither discussion nor guidance on the matter.

\section{Research subjects and informed consent}

The guidelines pay little attention to recruitment of research subjects apart from abandoning, after considerable criticism, the proposal in the draft guidelines that there should be an absolute ban on nontherapeutic research on children under 18 years old. The final version stresses that all the safeguards for therapeutic research with children must also apply to non-therapeutic research, together with the requirement that "the child must be subject to no more than minimal risk as a result of his or her participation." The phase "minimal risk" is ambiguous and could mean something like "the most trivial of procedures"a phrase used with regard to consent elsewhere in the guidelines - or "an extremely low chance of serious harm." Again, clarification is required.

Ethics committees are concerned that research subjects are given appropriate information to permit a choice about whether to participate, but there has been little guidance about what is appropriate. The law has offered little help since no case has turned on the provision of information in the research setting, and the general principles concerning consent to treatment adopted in the Sidaway case ${ }^{10}$ may not be held to apply to the framework of research. Now, however, the guidelines seem to offer a clear policy: "Volunteers must be told in advance of all known risks. ..." The word volunteer should be taken to mean all research subjects and not just healthy participants, which would restrict the policy to non-therapeutic research. However, full implementation of the guidelines would take us far beyond the current standards of disclosure, and some would argue that giving full information to everyone carries its own risks.

The apparent clarity of the guidelines becomes blurred when the phrase "all known risks" is considered. Although the concept might be useful in relation to established treatments, the inherent uncertainties of the research setting make it difficult to interpret. Should the phrase be understood to mean suspected risks, or risks associated with different drugs of a similar class, or what?

\section{Compensation}

The guidelines insist that all research subjects must be told of the arrangements for compensation if they suffer as a result of their participation, and the possible difficulties in obtaining compensation. It is clearly desirable that subjects are aware of these matters, but we question whether doctors can keep up to date with the various legal aspects of negligence liability and explain them clearly to all research subjects.

There would be some improvement if all drug companies, whether British or foreign, accepted responsibility in line with the Association of the British Pharmaceutiçal Industry guidelines, which provide a form of no fault compensation. Since availability of compensation is clearly an ethical issue which should concern the committees, it would be appropriate to make acceptance of the association's guidelines a condition for committee approval. This would go some way towards ameliorating for the research subject the harsh world of the law of negligence.

\section{Monitoring research}

Neither the 1975 circular nor the earlier draft of the present guidelines gave much attention to whether or how the conduct of research might be monitored. There is no suggestion that committees should ascertain whether consent to research is recorded in the notes, whether the general practitioner is notified of their patient's participation, and so on. Only if there is significant deviation from the original protocol, or unusual or unexpected results which might raise questions about the safety of research, is the researcher expected to notify the committee of what has happened. Committees do not, of course, have the resources to monitor the conduct of research projects, not even the simple procedures envisaged above; but the absence of such a duty raises questions about the efficacy of the regulatory mechanism as a whole.

The recent report by Julia Neuberger for the King's Fund Institute ${ }^{11}$ puts great emphasis on the need for monitoring, and recommends spot checks on research in progress to ensure that it is being carried out in accordance with the approval given by the ethics committee. Some committees may wish to move in this direction even though the guidelines do not require it. ${ }^{12}$

\section{Multicentre studies}

The role of committees in reviewing protocols for multicentre studies has been much discussed, particularly whether a research team should have to obtain separate agreement from the committee in each district where the work is to be carried out. The guidelines manage to face both ways on this, in the same paragraph: "Each LREC is free to arrive at its own decision when considering a proposal which is planned 
to take place in more than one area" and "committees should arrive at a voluntary arrangement under which one LREC is nominated to consider the issue on behalf of all of them." If a tidy administrative solution is sought-and it is questionable whether that should have precedence over local autonomy - it is surprising that no mention is made of the possible role of a national research ethics committee in authorising multicentre studies conducted under the auspices of, for example, the Medical Research Council or Cancer Research Campaign.

The guidelines will also produce other frustrations for those planning multicentre studies. Some committees have their own application forms which must be completed and returned together with the protocol under consideration. Inevitably, these forms have not been standardised, with the result that many different forms may be required for the same study. The department should produce standard application forms for all proposed research projects, especially as this would also help maintain the register of proposed research which all ethics committees must now keep.

Lack of attention to practical detail is seen throughout the guidelines which, despite an assertion to the contrary in the accompanying circular, also fail to consider ethical principles governing the conduct of research. Perhaps it was never the department's intention to provide such a discussion. For whatever reason, those who serve on committees will have to seek ethical guidance elsewhere.

\section{Implementation}

District health authorities should have established committees operating in accordance with the guidelines by 1 February 1992. Many existing committees may assume that they have been operating largely within the spirit of the guidelines and that any changes in their procedures will be minimal. But this assumption ignores the new requirement that it is only the responsible NHS body which can approve a research proposal and that the committee's role is to offer independent advice on ethical issues. There is, however, a good argument for more public discussion of the costs and priorities in medical research. The old system of committees approving research purely on the basis of ethics was entirely ineffective in this respect, but the new arrangements place no obligation on the district health authority or other NHS bodies to discuss the issues in public. Ironically, the guidelines require an annual report from the committee in its advisory role but envisage no form of public report from the body that will actually decide whether research proposals will go ahead.

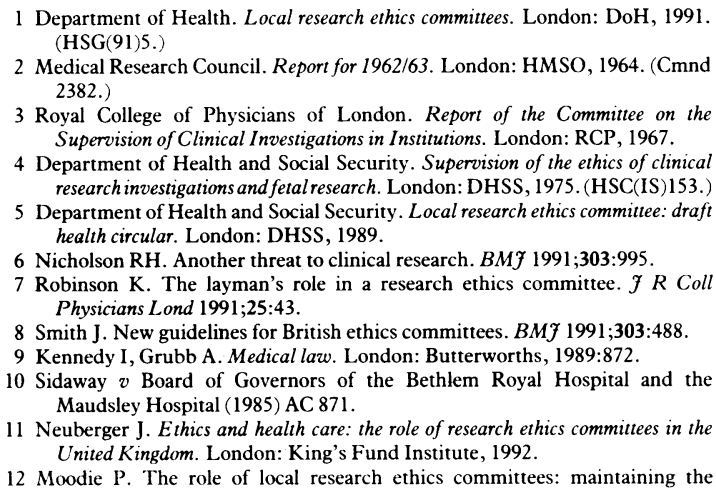

2 Medical Research Council. Report for 1962/63. London: HMSO, 1964. (Cmnd 2382.)

3 Royal College of Physicians of London. Report of the Committee on the Supervision of Clinical Investigations in Institutions. London: RCP, 1967.

4 Department of Health and Social Security. Supervision of the ethics of clinical research investigations and fetal research. London: DHSS, 1975. (HSC(IS)153.) 5 Department of Health and Social Security. Local research ethics committee: drafi health circular. London: DHSS, 1989.

6 Nicholson RH. Another threat to clinical research. BMf 1991;303:995.

7 Robinson K. The layman's role in a research ethics committee. $\mathcal{J} R$ Coll Physicians Lond 1991;25:43.

8 Smith J. New guidelines for British ethics committees. BMF 1991;303:488

9 Kennedy I, Grubb A. Medical law. London: Butterworths, 1989:872.

10 Sidaway $v$ Board of Governors of the Bethlem Royal Hospital and the Maudsley Hospital (1985) AC 871 .

11 Neuberger J. Ethics and health care: the role of research ethics committees in the United Kingdom. London: King's Fund Institute, 1992.

2 Moodie P. The role of local research ethics committees: maintaining the pressure for improvement. BMJ 1992;304:1129-30.

(Accepted 11 February 1992)

\section{Hairmyres Hospital, East} Kilbride G75 8RG

Anthony J Pelosi, consultant psychiatrist

University Hospital of South Manchester, Manchester M20 8LR Louis Appleby, senior lecturer in psychiatry

Correspondence to: Dr Pelosi.

BMF 1992;304:1295-8

\section{For Debate}

\section{Psychological influences on cancer and ischaemic heart disease}

\author{
Anthony J Pelosi, Louis Appleby
}

For over a decade two academic psychologists, Professor H J Eysenck and Professor R GrossarthMaticek, have reported a programme of research on the cause, prevention, and treatment of fatal diseases. Their main hypotheses are that particular personality traits increase the incidence of cancers and vascular diseases and that unhealthy personalities can be altered by psychological therapies, with a consequent reduction in death rates.

Unfortunately, descriptions of their methods, analyses, and results have been patchy and scattered widely in conference proceedings or in obscure or unrefereed journals and books..$^{1-6}$ Now, however, Eysenck and Grossarth-Maticek have published more detailed accounts in the widely read journal Behaviour Research and Therapy. ${ }^{78}$ These long awaited papers contain some of the most remarkable claims ever to appear in a refereed scientific journal and it is difficult for anyone interested in the influence of psychological factors on physical illnesses to continue to ignore this work.

\section{The investigations}

The papers are based mainly on three aetiological investigations and several intervention studies. The first was an epidemiological study in the town of Crvenka in Yugoslavia. In 1965 a total of 1353 people were recruited, consisting of the oldest persons in randomly selected households plus 345 people who were considered "at high psychosomatic risk," the definition of which was "based on chronic hopelessness due to withdrawing objects or chronic anger due to disturbing objects." Almost all subjects were aged between 48 and 68 years $5 ; 71 \%$ were male, apparently because of selection of the oldest member of each household, although a later paper explained that the sex ratio was due to "the cultural preponderance of males, which for the sake of a good atmosphere for investigation, we did not try to overcome." serious illnesses and causes of death were determined over the next 11 years. This follow up was $100 \%$ successful. ${ }^{+}$

In another study a random sample of 1026 people, mostly aged 40-60 years, was investigated in Heidelberg, Germany, between 1972 and 1982. Participants were asked to nominate friends and relatives who were "highly stressed," and these 1537 subjects, mainly aged 42-63 years, became the sample for a third cohort study. 5

The investigators selected individuals from these and other Heidelberg surveys who were, in their view, at particular risk of developing either neoplastic or vascular diseases and randomly allocated them to preventive psychological interventions with follow up over the next seven to 13 years. ${ }^{8}$ The various 